\title{
Efecto del protocolo de inducción hormonal en la espermiación y las características seminales de yaque (Leiarius marmoratus)
}

\author{
Effect of hormone induction protocol in the spermiation and \\ seminal characteristics of yaque (Leiarius marmoratus)
}

\section{Efeito do protocolo de indução hormonal sobre a spermiação e as características do sêmen do yaque (Leiarius marmoratus)}

\author{
Diego A Riveros-Pinilla ${ }^{\text {*}}$; Juan A Ramírez-Merlano ${ }^{2 *}$; Leydy Y. Sandoval-Vargas ${ }^{3 *}$; \\ Tatiana M Mira-López ${ }^{4}$; Pablo E Cruz-Casallas ${ }^{5}$; Víctor M. Medina-Robles ${ }^{6}$
}

1 MVZ;

2 Profesional en Acuicultura, MSc;

3 Ingeniera Pecuaria;

4 Z, MSc, PhD;

5 MVZ, MSc, PhD;

$6 \mathrm{MVZ}, \mathrm{MSc}$

* Grupo de Investigación sobre Reproducción y Toxicología de Organismos Acuáticos GRITOX, Instituto de Acuicultura, Universidad de los Llanos, Villavicencio, Colombia. Km 12 vía Puerto López, vereda Barcelona.

Email: vmmedinarobles@unillanos.edu.co

\section{Resumen}

El yaque Leiarius marmoratus, es una especie de siluriforme nativo con gran potencial para la diversificación de la acuicultura colombiana; sin embargo, la espermiación en cautiverio aún presenta dificultades debido al bajo volumen seminal liberado con los protocolos de inducción tradicionales. En consecuencia, el objetivo fue evaluar los efectos de cinco protocolos de inducción hormonal, basados en Extracto de Hipófisis de Carpa (EHC), sGnRHa + domperidona (OVAPRIM $R$ ) y Gonadotropina Coriónica Humana (GHC) (FERTIVET\&), sobre la calidad y volumen seminal de la especie. Fueron seleccionados 18 machos sexualmente maduros de 54,4 $\pm 2,2 \mathrm{~cm}$ de longitud total y 1,6 \pm 0,1 kg peso corporal. Se evaluaron 5 tratamientos, así: T1: 0,25 ml/kg (0 h) y $1 \mathrm{ml} / \mathrm{kg}$ de OVAPRIM® (12 h); T2: $1 \mathrm{mg} / \mathrm{kg}$ de EHC (0 h) y $3 \mathrm{mg} / \mathrm{kg} \mathrm{de} \mathrm{EHC} \mathrm{(12}$ h); T3: dosis única de $200 \mathrm{Ul} / \mathrm{kg}$ de GCH; T4: $3 \mathrm{mg} / \mathrm{kg}$ de EHC (0 h) y $200 \mathrm{Ul} / \mathrm{Kg}$ de GHC (8h); T5: $1 \mathrm{ml} / \mathrm{kg}$ de OVAPRIM (0h) y $200 \mathrm{UI} / \mathrm{kg}$ de GHC (8h); Control: $1 \mathrm{ml}$ de suero fisiológico. El semen se colecto $6 \mathrm{~h}$ después de aplicada la última dosis hormonal. El T1 y T5 mostraron un volumen mayor $(\mathrm{p}<0,05)(5,4 \pm 1,1 ; 4,8 \pm 1,2 \mathrm{ml})$ cuando comparados con T2 $(3,1 \pm$ $1 \mathrm{ml})$, T3 $(2,3 \mathrm{ml}) \mathrm{T} 4(2,4 \pm 1,1 \mathrm{ml})$ y el control $(0,2 \mathrm{ml})$. En cuanto a la movilidad y tiempo de activación, T1 y T5 presentaron los mejores resultados (90 $\pm 0 \%$ y $54,2 \pm 7 \mathrm{~s} ; 90 \pm 0 \%$ y 52,9 $\pm 3,8 \mathrm{~s}$, respectivamente). La concentración espermática fue mayor $(p<0,05)$ en T2 y T5 $\left(1,275 \pm 322 ;\right.$ y 1,261 $\left.\pm 225 \mathrm{sptz} \times 10^{3} / \mu \mathrm{l}\right)$ comparadas con los otros tratamientos. Se concluye que la inducción hormonal con OVAPRIM® combinada con HCG, son efectivas para la producción seminal en la especie.

Palabras clave: bagres, domperidona, extracto hipófisis de carpa, gonadotropina coriónica humana, semen. 


\begin{abstract}
The yaque Leiarius marmoratus is specie of native silurid, with great potential for the diversification of the colombian aquaculture; however, the spermiation in captive yet presents difficulties due to the low seminal volume released with traditional induction protocols. In consequence, the aim of this study was to evaluate the effects of five protocols of hormonal induction based on carp pituitary extract (EHC), sGnRHa + domperidone (OVAPRIM®) and human chorionic gonadotropin (GHC) (FERTIVETR) on seminal quality and volume of the specie. Were selected 18 sexually mature males of 54,4 $\pm 2,2 \mathrm{~cm}$ in total length and 1,6 $\pm 0,1 \mathrm{~kg}$ body weight. 5 treatments were evaluated so: T1:0,25 ml/kg $(0 \mathrm{~h})$ and $1 \mathrm{ml} / \mathrm{kg}$ of OVAPRIM® (12 h ); T2: $1 \mathrm{mg} / \mathrm{kg}$ of EHC (O h) and $3 \mathrm{mg} / \mathrm{kg}$ of EHC (12 h); T3 : single dose of $200 \mathrm{UI} / \mathrm{kg} \mathrm{HCG}$; T4: $3 \mathrm{mg} / \mathrm{kg}$ of EHC (0 h) and $200 \mathrm{UI} / \mathrm{kg} \mathrm{GHC}(8 \mathrm{~h})$; T5: $1 \mathrm{ml} / \mathrm{kg}$ of OVAPRIM® (Oh) and $200 \mathrm{U.I} / \mathrm{kg}$ of GHC (8h); Control: $1 \mathrm{ml}$ of saline solution. Semen was obtained 6 hours after the last dose hormone. T1 and T5 showed a larger volume $(p<0.05)(5,4 \pm 1,1,4,8 \pm 1,2$ $\mathrm{ml})$ compared to T2 $(3,1 \pm 1 \mathrm{ml})$, T3 $(2,3 \mathrm{ml}) \mathrm{T} 4(2,4 \pm 1.1 \mathrm{ml})$ and control $(0.2 \mathrm{ml})$. Regarding motility and activation time, $\mathrm{T} 1$ and T5 had the best results (90 $\pm 0 \%$ and $54,2 \pm 7 \mathrm{~s} ; 90 \pm 0 \%$ and 52,9 $\pm 3,8 \mathrm{~s}$, respectively). The sperm concentration was higher in $(p<0,05)$ T2 and T5 $(1,275 \pm 322, \pm 225$ and 1,261 sptz $\times 103 /$ ul) compared with the other treatments. In conclusion, hormonal induction with OVAPRIM® combined with HCG, are effective for the seminal production in the specie.
\end{abstract}

Key words: carp pituitary extract, catfish, domperidone, human chorionic gonadotropin, spermatozoa.

\begin{abstract}
Resumo
O yaque Leiarius marmoratus, é uma espécie siluriforme nativa com grande potencial para a diversificação da aquicultura colombiana; No entanto, a spermiação em cativeiro ainda apresenta dificuldades devido ao baixo volume seminal liberado com protocolos de indução tradicionais. Por conseguinte, o objetivo foi avaliar os efeitos de protocolos de indução de cinco hormonais base de extrato de hipófise de carpa (EHC), sGnRHa + domperidona (Ovaprim®) e Gonadotropina coriónica humana (GHC) (FERTIVET®) sobre a qualidade e volume seminal das espécies. Foram Selecionados 18 machos sexualmente maduros de 54,4 2,2 cm de comprimento total e 1,6 \pm 0,1 kg de peso corporal. Foram avaliados cinco tratamentos: T1: 0,25 ml / kg (0 h) e $1 \mathrm{ml} / \mathrm{kg}$ de OVAPRIM® (12 h); T2: $1 \mathrm{mg} / \mathrm{kg}$ de EHC (0 h) e $3 \mathrm{mg} / \mathrm{kg}$ de EHC (12 h); T3: dose única de $200 \mathrm{UI} / \mathrm{kg}$ de HCG; T4: 3 mg / kg de EHC (0 h) e $200 \mathrm{UI} / \mathrm{kg}$ de GHC (8h); T5: 1 ml / kg de OVAPRIM® (Oh) e 200 UI / kg de GHC (8h); Controle: $1 \mathrm{ml}$ de solução salina fisiológica. O sêmen foi coletado $6 \mathrm{~h}$ após a última dose hormonal foi aplicada. T1 e T5 mostraram um volume maior $(\mathrm{p}<0,05)(5,4 \pm 1,1,4,8 \pm 1,2 \mathrm{ml})$ quando comparado com T2 (3,1 $\pm 1 \mathrm{ml})$, T3 $(2,3 \mathrm{ml}) \mathrm{T} 4(2,4 \pm 1,1 \mathrm{ml})$ e controle $(0,2 \mathrm{ml})$. Em relação à mobilidade e ao tempo de ativação, T1 e T5 apresentaram os melhores resultados ( $90 \pm 0 \%$ e 54,2 \pm 7 s, $90 \pm 0 \%$ e 52,9 $\pm 3,8$ s, respectivamente). A concentração de esperma foi maior ( $p<0,05)$ em T2 e T5 (1275 \pm 322 e $1261 \pm 225$ sptz x 103 / $\mu$ l) em comparação com os outros tratamentos. Conclui-se que a indução hormonal com OVAPRIM® combinada com HCG, é efetiva para a produção seminal nas espécies.
\end{abstract}

Palavras-chave: carpa extrato de pituitária, domperidona, gonadotrofina coriônica humana, peixe gato, sêmen.

\section{Introducción}

En Colombia se ha generado un creciente interés por cultivar e investigar especies de peces del orden Siluriformes con potencial para la acuicultura. Sin embargo, la limitada e inestable producción de alevinos debido a la marcada estacionalidad reproductiva de las especies y por las altas tasas de canibalismo durante las primeras etapas de vida ha dificultado su producción a escala comercial. Al respecto, varios estudios han sido desarrollados principalmente en Pseudoplatystoma orinocoense, Leiarius marmoratus y Pseudoplatystoma metaense. L marmoratus (Gill, 1870) es una especie de gran importancia comercial para las cuencas de los ríos Orinoco y Amazonas. En condiciones de cautiverio, reporta buen crecimiento en los primeros estadios de vida (Cruz-Casallas et al., 2008), un significativo desempeño productivo de su cultivo en estanques en tierra (Cruz-Casallas et al., 2010), así como un filete de excelente calidad nutricional (Cruz-Casallas et al., 2014), lo anterior atribuido a el hábito alimenticio omnívoro (Santos et al., 2006), que diferencia esta es- pecie de la mayoría de bagres. Leiarius marmoratus es una alternativa para fortalecer la acuicultura nacional, no obstante, similar a otros silúridos presenta disfunciones reproductivas cuando es mantenido en cautiverio (Zohar y Mylonas, 2001).

En general, en la mayoría de especies de peces los machos se adaptan mejor que las hembras a las condiciones de cultivo a las que son sometidos, y son capaces de producir gametos viables (Araujo et al., 2014), no obstante, es necesario administrar hormonas exógenas para inducir la espermiación final y aumento del volumen seminal. Los silúridos, en su mayoría responden positivamente a protocolos de inducción hormonal. Sin embargo, un escaso números de bagres como los Clarias gariepinus (Viveiros et al., 2002), Ictalurus furcatus (Gima et al., 2013), Pimelodus britskii (Damasceno et al., 2015), Leiarius marmoratus (Araújo et al., 2014) y Sorubim y lima (Shibatta et al., 2011) liberan mínimo volumen seminal mediante estrujamiento manual, comparado con el volumen obtenido directamente de los testículos. Varios factores han sido atribuidos 
como causa de este fenómeno. Según Damasceno et al., (2015), la morfología y ubicación anatómica de los testículos así como el protocolo de inducción hormonal y la fase de la estación reproductiva son determinantes en Pimelodus britskii. Desde otra perspectiva, Araújo et al., (2014), relaciona esta deficiencia en $L$. marmoratus con la falta de domesticación de los reproductores. Por consiguiente, es necesario realizar estudios específicos para cada especie.

Sustancias inductoras como Extracto de Hipófisis de Carpa (EHC) y análogos de la hormona liberadora de gonadotropina $\left(\mathrm{GnRH}, \mathrm{LHRH}\right.$, Ovaprim $\left.{ }^{\circledR}\right)$ han dado resultados positivos en la inducción a la maduración final y ovulación de las especies nativas (Atencio, 2001). Sin embargo, el EHC presenta algunas desventajas, como su variabilidad en la cantidad y calidad de hormonas presentes y su alto costo en el comercio. En contraste la hormona liberadora de gonadotropina de salmón con un bloqueador de los receptores de D2 de dopamina, Ovaprim ${ }^{\circledR}$ presenta ventajas, como la no generación de respuesta inmunológica, induce la liberación de $\mathrm{GtH}$ endógeno desencadenando desde un nivel superior los procesos de maduración, ovulación, desove y espermiación.

En consecuencia, este trabajo se realizó con el fin de evaluar los efectos de cinco protocolos de inducción hormonal basados en Extracto de Hipófisis de Carpa (EHC), sGnRHa + domperidona (OVAPRIM $®)$ y Gonadotropina Coriónica Humana $(\mathrm{GCH})$, sobre la respuesta a la espermiación y la calidad seminal en yaque.

\section{Materiales y métodos}

\section{Área de estudio}

El estudio se llevó a cabo en el Instituto de Acuicultura de la Universidad de los Llanos, localizado a $4 \mathrm{~km}$ de la ciudad de Villavicencio, Colombia. La estación piscícola se encuentra a una altitud 418 m.s.n.m, presenta una pluviosidad de $4050 \mathrm{~mm}$ anuales, temperatura ambiental media de $25^{\circ} \mathrm{C}$, temperatura promedio del agua de $27^{\circ} \mathrm{C}$ y humedad relativa del $75 \%$.

\section{Material biológico}

Se utilizaron 18 machos adultos de 1,6 \pm 0,1 $\mathrm{kg}$ de peso corporal y $54,4 \pm 2,2 \mathrm{~cm}$ de longitud total. Los reproductores fueron alojados en estanques de tierra de $372 \mathrm{~m}^{2}$ a una densidad promedio de $240 \mathrm{~g} / \mathrm{m}^{2}$, donde fueron alimentados con concentrado comercial del $25 \%$ de PB.
Los ejemplares fueron seleccionados teniendo en cuenta su desarrollo corporal y condición de madurez sexual, evidenciada por la emisión de un fluido transparente y oleoso a través de la papila urogenital ante presión cráneo-caudal del abdomen. Los reproductores seleccionados se trasladaron a piletas circulares de manejo de $7 \mathrm{~m}^{3}$, donde permanecieron por lo menos 24 horas antes de iniciar el proceso de inducción y permanecieron allí hasta el final del experimento. Se registró el peso $(\mathrm{kg})$, longitud total y la longitud estándar $(\mathrm{cm})$ de cada individuo.

\section{Inducción hormonal}

De acuerdo a ensayos preliminares, fueron evaluados cinco tratamientos, utilizando Fertivet ${ }^{\circledR}$ - Gonadotropina Coriónica Humana $(\mathrm{GCH})$, Extracto de Hipófisis de carpa (EHC) y una mezcla comercial de análogo superactivo de hormona liberadora de gonadotropina con un bloqueador de los receptores D2 de dopamina, sGnRH + domperidona (OVAPRIM®). Los tratamientos consistieron en aplicación vía intramuscular de: T1: $0,25 \mathrm{ml} / \mathrm{kg}$ de OVAPRIM ${ }^{\circledR}(0 \mathrm{~h})$ y $1 \mathrm{ml} / \mathrm{kg}$ de OVAPRIM ${ }^{\circledR}(12 \mathrm{~h}) ; \mathrm{T} 2: 1 \mathrm{mg} / \mathrm{kg}$ de EHC (0 h) y $3 \mathrm{mg} / \mathrm{kg}$ de EHC (12 h); T3: dosis única de 200 U.I/kg de GCH; T4: $3 \mathrm{mg} / \mathrm{kg}$ de EHC (0 h) y $200 \mathrm{U.I} / \mathrm{Kg}$ de GCH (8h); T5: $1 \mathrm{ml} / \mathrm{kg}$ de OVA (0h) y $200 \mathrm{U.I} / \mathrm{kg}$ de GCH (8h); el grupo control consistió en la aplicación de suero fisiológico $(\mathrm{NaCl} 0,9 \%)$, siguiendo el mismo cronograma de los individuos tratados hormonalmente.

\section{Obtención del semen}

Transcurridas 6 horas después de aplicada la última dosis en cada uno de los cinco tratamientos se extrajo el semen. Previo a la extracción de los gametos, los animales fueron tranquilizados por inmersión en una solución de 2-fenoxietanol (300 ppm, Sigma Chemical Co., St. Louis, Missouri, USA), durante 5 min. Inmediatamente después de la pérdida del eje de nado, los animales fueron extraídos y secados en la región abdominal, aplicando una leve presión para provocar la expulsión de orina y un masaje cráneo-caudal de la pared celómica para provocar la emisión del semen. Este último fue colectado en tubos de vidrio aforados para determinar directamente el volumen, teniendo el cuidado de evitar contaminación con bilis, orina o heces, ya que varios estudios han mostrado disminución en algunos parámetros como son: porcentaje de movilidad, velocidad espermática, frecuencia de remolino y posible activación de la célula espermática (Viveiros et al., 2010). El fluido extraído fue llevado inmediatamente al Laboratorio de Reproducción donde se mantuvo 
a una temperatura de $26^{\circ} \mathrm{C}$ hasta terminar las evaluaciones espermáticas.

\section{Evaluación seminal}

La concentración espermática se determinó dentro de las 8 h siguientes a la colección del semen por recuento en cámara de Neubauer, previa dilución del semen 1:4000, con solución salina formolada $(0,9 \%$ de $\mathrm{NaCl}$ y formol al 3 \%) (Ramírez-Merlano et al., 2011). La cámara se mantuvo en atmósfera húmeda durante al menos 10 min antes del conteo de las células (40X). Para evaluar la movilidad masal (MM) y el tiempo de activación espermática (TA) se realizó una dilución 1:20 (20 $\mu \mathrm{L}$ de semen + $180 \mu \mathrm{L}$ de agua), sobre una lámina excavada (profundidad 1 - 1,2 mm, Micro-Slides Premiere, China) dispuesta en un microscopio óptico (10 X de magnificación), la cual se activó con agua (180 $\mu \mathrm{L})$. Desde la activación espermática hasta el momento en que el $90 \%$ de los espermatozoides cesaron el movimiento, se registró el tiempo de activación con un cronometro y se expresó en segundos.

\section{Análisis estadístico}

Los resultados fueron expresados como media \pm error estándar de la media (SEM). Para determinar los efectos de los tratamientos, los resultados fueron sometidos a un análisis de varianza de una vía (ANOVA), previa validación de los supuestos de homogeneidad de varianza y normalidad de los datos, seguido de una prueba de comparación de Tukey-Kramer, con un nivel de significancia de $p<0,05$. Los datos fueron anali- zados con el software SAS versión 8.02 para Windows (1999-2001 por SAS Institute Inc, Cary, NC. USA).

\section{Resultados}

El porcentaje de respuesta a los inductores fue del $100 \%$ en todos los tratamientos, excepto en Gonadotropina Coriónica Humana (T3), donde sólo el 33\% de los individuos inducidos libero semen. Las muestras seminales presentaron coloración blanquecina. Los individuos tratados con suero fisiológico (grupo control) liberaron mínimas cantidades de un líquido casi transparente sin presencia de espermatozoides, por lo que fueron excluidos de los análisis.

La tabla 1 muestra los valores medios de las características seminales obtenidas con los diferentes tratamientos, distribuyéndose aproximadamente 3 animales por tratamiento. El volumen seminal $(\geq 4,8 \mathrm{ml})$ la movilidad masal $(90 \%)$ y tiempo de activación (>50s) fue significativamente mayor en los machos tratados con Ova-

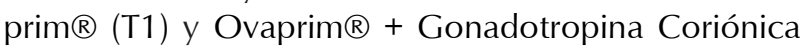
Humana (T5), en tanto que los machos tratados con solo Gonadotropina Coriónica Humana (T3) presentaron menores valores de estos parámetros $(0,73 \mathrm{ml}$, $13,3 \%$ y $16 \mathrm{~s}$, respectivamente).

Por su parte, el tiempo de activación fue igual en todos los tratamientos, presentando un valor mínimo de $16 \pm 28$ s y máximo de 54,2 \pm 7 s. Finalmente, la concentración espermática, fue mayor en los machos inducidos con Extracto de Hipófisis de Carpa

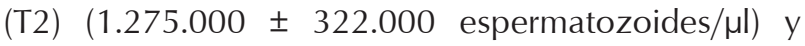
Ovaprim ${ }^{\circledR}+$ Gonadotropina Coriónica Humana (T5)

Tabla 1. Características seminales de yaque, tratados con cinco protocolos hormonales para inducir la espermiación. Letras diferentes entre filas indican diferencias significativas entre tratamientos $(p<0,05)$. Valores mostrados corresponden a la media \pm SEM.

\begin{tabular}{|l|c|c|c|c|}
\hline \multicolumn{1}{|c|}{ Tratamiento } & Volumen $(\mathbf{m L})$ & $\begin{array}{c}\text { Movilidad } \\
\text { Masal } \\
\mathbf{( \% )}\end{array}$ & $\begin{array}{c}\text { Tiempo de } \\
\text { Activación } \\
\mathbf{( s )}\end{array}$ & $\begin{array}{c}\text { Concentración } \\
\text { Espermática } \\
\left.\mathbf{( 1 0}^{\mathbf{3}} \mathbf{s p t z} / \boldsymbol{\mu l}\right)\end{array}$ \\
\hline T1 sGnRH + Domperidona & $5,4 \pm 1,1^{\mathrm{b}}$ & $90 \pm 0^{\mathrm{b}}$ & $54,2 \pm 7$ & $896,7 \pm 329^{\mathrm{ab}}$ \\
\hline T2 Extracto de Hipófisis de Carpa & $3,0 \pm 0,8^{\mathrm{ab}}$ & $76,6 \pm 15,2^{\mathrm{b}}$ & $47,1 \pm 3$ & $1,275 \pm 372^{\mathrm{b}}$ \\
\hline T3 Gonadotropina Coriónica Humana & $0,73 \pm 1,2^{\mathrm{a}}$ & $13,3 \pm 23^{\mathrm{a}}$ & $16 \pm 28$ & $271,6 \pm 470^{\mathrm{a}}$ \\
\hline $\begin{array}{l}\text { T4 } \text { Extracto de Hipófisis de carpa }+ \\
\text { Gonadotropina Coriónica Humana }\end{array}$ & $2,5 \pm 1,4^{\mathrm{ab}}$ & $70 \pm 26^{\mathrm{b}}$ & $48,7 \pm 5$ & $895 \pm 178^{\mathrm{ab}}$ \\
\hline $\begin{array}{l}\text { T5 } \text { sGnRH + Domperidona + } \\
\text { Gonadotropina Coriónica Humana }\end{array}$ & $4,8 \pm 1,2^{\mathrm{b}}$ & $90 \pm 0^{\mathrm{b}}$ & $52,9 \pm 4$ & $1,281 \pm 225^{\mathrm{b}}$ \\
\hline
\end{tabular}


$(1.281 .000 \pm 225.000$ espermatozoides/ $\mu \mathrm{l})$ mientras que el menor valor $(2.716 .000 \pm 470.000)$ fue obtenido en los machos inducidos con Gonadotropina Coriónica Humana $(p<0,05)$.

\section{Discusión}

Según la literatura algunos silúridos, como L. marmoratus, Clarias gariepinus (Viveiros et al., 2002; Bruzka, 2003), Ictalurus punctatus (Christensen y Tiersch 2005), Zungaro jahu (Viveiros et al., 2009) necesitan de tratamiento hormonal para inducir la espermiación. Destacandose en este estudio los resultados con OVAPRIM ${ }^{\circledR}$ (sGnRHa + domperidona), observándose un volumen seminal $\geq 4,8 \mathrm{ml}$, siendo superior al reportado por Mira-López et al., (2010) (2,84ml) y por Araújo et al., (2014) $(1,45 \mathrm{ml})$ para la misma especie. Lo que permitirá obtener un semen con alta viabilidad para la crioconservación.

El T1 y T5 mostraron los mayores valores de volumen $(5,4 \pm 1,1 ; 4,8 \pm 1,2 \mathrm{ml})$, movilidad y tiempo de activación ( $90 \pm 0 \%$ y $54,2 \pm 7$ s; $90 \pm 0 \%$ y $52,9 \pm 3,8 \mathrm{~s}$, respectivamente) con respecto a los otros tratamientos y al grupo control. Lo que permite inferir que estos tratamientos al aumentar el volumen seminal durante el proceso de la espermiación inducida hormonalmente no interfiere en la calidad seminal. Con respecto a la concentración espermática fue mayor en T2 y T5

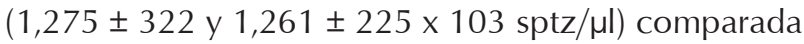
con los otros tratamientos.

Es apropiado citar la problemática de colección de esperma por estrujamiento en estas especies de bagres que no podría ser resuelto mediante la administración de hormonas exógenas como GtH o GnRHa combinadas con antagonistas de la dopamina (Mylonas et al., 2010). No obstante, las terapias hormonales empleadas en este estudio permitieron obtener por primera vez muestras seminales de yaque (Leiarius marmoratus) con características adecuadas para realizar procedimientos de fertilización in vitro, así como someterlas a procesos de crioconservación.

Los valores de movilidad masal de semen fresco en silúridos son superiores a $80 \%$, aunque, Brand et al., (1996) reportan valores de movilidad masal de 90,2 $\pm 4,7 \%$ para Pseudoplatystoma fasciatum. Igualmente, evaluaciones realizadas en otros silúridos como la barbilla (Rhamdia sebae c. f.) reportan una alta movilidad del semen fresco, 94,0 \pm 0,7\% (Villalobos-Sánchez y Osorio-Velandia, 2007) y $91,0 \pm 2,3 \%$ (Velasco-Santamaría et al., 2004). Se reporta para el bagre sapo (Rhamdia quelen) una movilidad masal del semen del 80.0 $\pm 1,0 \%$, mientras que Araújo et al., (2003) reportaron una movilidad masal promedio de $87,0 \%$ para Sorubim cuspicaudus, un poco más baja que la reportada anteriormente para los otros silúridos. Estudios anteriores sobre evaluación de las características seminales en yaque, realizadas por Mira et al., (2010), obtuvieron un volumen promedio de 2,7 $\pm 0,7 \mathrm{ml}$, menor al compararlo con el volumen promedio obtenido en este estudio $(5,4 \pm 1,1 \mathrm{ml})$, aunque el volumen encontrado por Brand (1996) en Pseudaplatystoma fasciatum fue significativamente alto en comparación con los reportados anteriormente $(24,5 \pm 21,7 \mathrm{ml})$.

Cabe señalar que la obtención del semen a partir del sacrificio de los machos, compromete los programas de selección y mejoramiento genético de estas especies, por lo tanto es importante lograr omitir la intervención de la técnica quirúrgica para conseguir programas de reproducción económicamente rentables (Viveiros et al., 2002).

Los tratamientos utilizados aumentaron significativamente $(p<0,05)$ la movilidad espermática y no afectaron su duración. En cuanto a la movilidad y tiempo de activación, T1 (OVAPRIM $®)$ y T5 (OVAPRIM® + GCH) presentaron los mejores resultados (90 $\pm 0 \%$ y 54,2 $\pm 7 \mathrm{~s} ; 90 \pm 0 \%$ y $52,9 \pm 3,8 \mathrm{~s}$, respectivamente). La concentración espermática fue mayor $(p<0,05)$ en T2 (EHC) y T5 (OVAPRIM $\left.{ }^{\circledR}+\mathrm{GCH}\right)(1,275 \pm 322 ;$ y 1,261 $\left.\pm 225 \mathrm{sptz} \times 10^{3} / \mu \mathrm{L}\right)$ comparadas con los otros tratamientos. Los valores hallados en los machos tratados con $\operatorname{EHC}(76,6 \pm 15,2 \%)$ fueron superiores a los encontrados en la misma especie con una dosificación menor de EHC $(40 \pm 34 \%)$ y fueron similares a los encontrados en Pseudoplatystoma sp. con el mismo inductor en dosis única (95 \%) (Rivera et al., 2009).

Han sido evaluados diferentes inductores de segunda y tercera generación para producir liberación y aumento del volumen seminal en varias especies de silúridos como Sorubim cuspicaudus (Muñoz y Martínez, 2003), Pseudoplatystoma fasciatum (Guarnizo, 2003) y Rhamdia sebae (Velasco-Santamaría et al., 2004), siendo los más utilizados extractos hipofisiarios heterologos de pez (carpa), análogos superactivos de la hormona liberadora de gonadotropinas (sGnRH-A) combinados con bloqueadores de dopamina y gonadotropina coriónica humana $(\mathrm{GCH})$, todos con resultados positivos. En C. gariepinus (Viveiros et al., 2009), evaluaron diferentes protocolos con base en extracto de hipófisis de carpa y de Clarias, mGnRHa con Pimozide y $\operatorname{OVAPRIM}^{\circledR}$, de manera individual y en varias combinaciones. En aquellos tratamientos donde lograron obtener semen por medio de estrujamiento, este fue en reducida cantidad y de inferior calidad cuando 
se comparó con el semen extraído directamente del testículo (Viveiros et al., 2002).

En conclusión los resultados del presente estudio permiten sugerir que la inducción hormonal con sGnRH

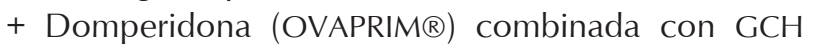
(FERTIVET $囚)$, son efectivas para la inducción hormonal de Leiarius marmoratus sin afectar su calidad seminal que permitirán obtener tasas de fertilidad satisfactorias en la reproducción de la especie bajo condiciones de cautiverio.

\section{Agradecimientos}

A la Dirección General de Investigaciones de la Universidad de los Llanos por el apoyo financiero (proyecto Código FCARN-12-2014), a los integrantes, jóvenes investigadores y Estudiantes Pasantes de Investigación (EPI) del Grupo GRITOX quienes apoyaron en las distintas etapas del desarrollo del mismo. Así como, el agradecimiento al Instituto de Acuicultura de la Universidad de los Llanos por el apoyo logístico para la realización de este estudio.

\section{Referencias}

Araújo J, Streit Jr, Danilo P, Ribeiro JS, Martins EF, Souza FN, Oliveira CA, Ricardo PR, Lopera-Barrero NM, Povh JA. Ovopel and Carp Pituitary Extract as Spawning Inducers in Males of the Amazon Catfish Leiarius marmoratus (Gill, 1970). Brazilian Archives of Biology and Technology, 2014;57(6):882-886

Araújo H. Cordero W. Rugeles C. y Atencio V. 2003. Evaluación de las características seminales de Blanquillo Sorubim cuspicaudus inducido con ovaprim ${ }^{\circledR}$. Revista Colombiana de Ciencias Pecuarias, 16 (suplemento): 78p.

Atencio GV. Producción de alevinos de especies nativas. Rev MVZ Córdoba; 2001;6(1):9-14.

Bobe J, Labbé C. Egg and sperm quality in fish. Gen Comp Endocrinol. 2009;165(3):535-48.

Brand B. 1996. Caracterización y preservación del semen de bagre rayado (Pseudoplatystoma fasciatum, Linnaeus, 1766). Santa Fe de Bogotá D.C. Trabajo de grado (Biólogo Marino). Universidad Jorge Tadeo Lozano. Facultad de Biología Marina.

Brzuska E. Artificial propagation of African catfish (Clarias gariepinus): differences between reproduction effects after stimulation of ovulation with carp pituitary homogenate or GnRH-a and dopaminergic inhibitor. Czech J Anim Sci. 2003;48(5):181-190.

Castillo Jiménez AM, Ramírez Lesmes R, Rodríguez Pulido JA. 2003. Ensayos de reproducción y alevinaje en Yaque Leiarius marmoratus (Gill, 1870) (Pises: Siluriformes: Pimelodidae) en la Orinoquia Colombiana. En: VI Seminario. Internacional de Acuicultura I Congreso Nacional de Investigaciones Acuícolas. Bogotá: Universidad Nacional de Colombia.
Christensen JM, Tiersch TR. Cryopreservation of channel catfish sperm: effects of cryoprotectant exposure time, cooling rate, thawing conditions, and male-to-male variation. Theriogenology. 2005;63:2103-2112.

Cruz-Casallas N, Díaz-Olarte JJ, Marciales-Caro LJ, Pabón-Peña F, Medina Robles V, Cruz-Casallas P.E. Acondicionamiento a dieta seca de larvas de yaque (Leiarius marmoratus) obtenidas por reproducción artificial. Revista Colombiana de Ciencias Pecuarias. 2008;21:482.

Cruz-Casallas NE, Marciales-Caro LJ, Díaz-Olarte JJ, Murillo-Pacheco R, Medina-Robles VM, Cruz-Casallas PE. Desempeño productivo del yaque (Leiarius marmoratus Gill, 1870) bajo diferentes densidades de siembra en estanques en tierra. Revista Colombiana de Ciencias Pecuarias. 2010;23:325-335.

Cruz-Casallas N, Díaz-Olarte JJ, Marciales-Caro LJ, Pabón-Peña F, Medina Robles V, Cruz-Casallas P.E. Acondicionamiento a dieta seca de larvas de yaque (Leiarius marmoratus) obtenidas por reproducción artificial. Revista Colombiana de Ciencias Pecuarias. 2008;21:482.

Cruz-Casallas NE, Cruz-Casallas PE, Suárez-Mahecha H. Quality of catfish meat Leiarius marmoratus during frozen storage. Revista Orinoquia. 2014;18: 43-52.

Damasceno DZ, Krause RA, Adames MS, Neumann G, Gibathe A, Bombardelli RA, Romagosa E. Induced spermiation of Pimelodus britskii (Teleostei: Pimelodidae) during the reproductive period. Aquaculture Research. 2015;1-13.

Duarte M, Marbá N, Holmer M. Rapid domestication of marine species. Science. 2007;16:382-383.

Gima ME, Gima A, Hutson A, Chaimongkol A, Beam R, Perera DA, Dunham RA. Realized heritability and response to selection for fecundity, hatching rate and fry $/ \mathrm{Kg}$ for channel catfish females (Ictalurus punctatus) induced to ovulate and fertilized with blue catfish (Ictalurus furcatus) males for the production of hybrid catfish embryos. Aquaculture, 2013; (Suppl. 1) s36-s41:420421.

Guarnizo Pineda M. 2003. Caracterización seminal y ensayos preliminares de crioconservación de semen de bagre rayado (Pseudoplatystoma fasciatum - Linnaeus 1766). [Trabajo de Grado]. Palmira, Colombia: Universidad Nacional de Colombia.

Kossowski C, Madrid F. Observaciones de los estadios embrionario y larval del bagre rayado cabezón Pseudoplatystoma fasciatum (Linnaeus) 1766 (Pisces, Siluriformes). Biollania. 1991;8:915.

Kossowski C. 1999. Perspectivas del cultivo de bagre en Venezuela. En: Memorias $2^{\circ}$ Congreso Sur Americano De Acuicultura: 287297. Puerto La Cruz - Venezuela.

Linhart O, Billard R. Spermiation and sperm quality of European catfish (Silurus glanis) after implantation of GnRH analogs and injection of carp pituitary extract. J Appl Ichthyo. 1994;10:182188.

Maldonado-Ocampo JA, Vari RP, Usma JS. Checklist of the freswater fishes of Colombia. Biota Colombiana. 2008;9(2):143-237.

Mira-López TM, Medina-Robles VM, Cruz-Casallas PE. Evaluación de tres protocolos hormonales para la inducción de la esper- 
miación en yaque Leiarius marmoratus. Revista MVZ Córdoba. 2010;15(2):2070-2077.

Mira-López TM, Murillo-Pacheco R, Ramírez-Merlano JA, Otero-Paternina AM, Berruecos-Zapata BE, Medina-Robles VM, Cruz-Casallas PE. 2008. Ensayos preliminares de reproducción inducida de yaque Leiarius marmoratus con extracto de hipófisis de carpa. Memorias IV Congreso Colombiano de Acuicultura.

Mira-López TM, Medina-Robles VM, Cruz-Casallas PE. Morfología Testicular del yaque Leiarius marmoratus (Pisces: Siluridae) en estadio de madurez reproductiva.International Journal of Morphology, 2010;28(2):421-426.

Mylonas, C.C., Fostier, A. and Zanuy, S. Broodstock management and hormonal manipulations of fish reproduction. General and Comparative Endocrinology. 2010;165:516-534. doi: 10.1016/j.ygcen. 2009.03.007

Murillo-Pacheco R, Cruz-Casallas N, Ramirez-Merlano JA, Marciales-Caro L, Medina-Robles VM, Cruz-Casallas PE. Efecto del nivel de proteína sobre el crecimiento del Yaque Leiarius marmoratus (Gill, 1870) bajo condiciones de cultivo. Orinoquia, 2012;16(2):52-61.

Muñoz R, Martínez C. 2003. Reproducción inducida del blanquillo (Sorubim cuspicaudus Littmann, Burr \& Nass, 2000) con Ovaprim ${ }^{\circledR}$. Trabajo de pregrado. Montería (Col): Universidad de Córdoba

Ramírez-Merlano JA, Medina-Robles VM, Cruz-Casallas PE. Crioconservación seminal de bagre rayado Pseudoplatystoma metaense (Teleostei, Pimelodidae), bajo diferentes protocolos de congelación. Archivos de Medicina Veterinaria. 2011;43:135-144.

Ramirez-Gil H y Ajiaco-Martínez RE. Aspectos preliminares de la biología pesquera del yaque, Leiarius marmoratus (Gill, 1870) (Piscis: Siluriforrmes: pimelodidae) en la parte alta del rio Meta (Orinoquia Colombiana). Boletín científico. INPA. 1997;5:7587.

Rivera López EA. 2009. Evaluación de las características del semen de bagre rayado Pseudoplatystoma sp. (PISCES: SILURIDAE) obtenido con diferentes inductores hormonales. [Trabajo de Grado]. Fusagasugá, Colombia: Universidad de Cundinamarca.

Perchec P., Paxion C., Cosson J., Jeulin G., Fierviitle F. y Billard R. Initiatin of the carp spermatozoa motility and earty ATP reduction after milt contamination by urine, laboratoire de biologie de la reproducao et de development, Aquaculture. 1998;160:317328.
Rodríguez-Pulido, J.A., 2004. Aspectos reproductivos del nicuro Pimelodus blochii (Velenciennes, 1840) en cautiverio en la estación piscícola "La Terraza", INPA Villavicencio, Meta. Facultad de Ciencias. Pontificia Universidad Javeriana, pp. 82.

Rurangwa E, Kime DE, Ollevier F, Nash JP. The measurement of sperm motility and factors affecting sperm quality in cultured fish. Aquaculture, 2004;234:1- 28.

Santos G, Ferreira E, Zuanon J. 2006. Peixes comerciais de Manaus. IBAMA/AM; ProVárzea, 135.

Shibatta, OA., Novelli, JL, Dias, JH P, de Castro Britto SG, Caetano Filho, M. Reprodução em cativeiro do jurupecê Sorubim lima (Siluriformes, Pimelodidae) por meio de indução hormonal. Semina: Ciências Agrárias, 2011;32(1):363-372.

Solano Camelo MF, Urueña Bermeo FR. Evaluación comparativa del efecto del extracto pituitario de carpa (EPC) y gonadotropina coriónica humana (HCG) en la espermiación del yaque (Leiarius marmoratus). En: VI Seminario Internacional de Acuicultura. Bogotá: Rev Med Vet Zoot. 2007;244.

Velasco-santamaría Y.M., Arias-Castellanos J.A. y Cruz-Casallas P.E. 2004. Efecto de la inducción hormonal con extracto de hipófisis de carpa (EHC) sobre algunas características seminales de Rhamdia sebae c. f. Memorias II Congreso Colombiano de Acuicultura, X Jornada de Acuicultura IALL. 116-117p.

Villalobos-Sanchez MA, DM Osorio-Velandia. 2007. Ensayos preliminares de crioconservacion de semen de Barbilla (Rhamdia sebae c.f.). Facultad de Ciencias Agropecuarias y Recursos Naturales, Universidad de los Llanos, Villavicencio, Colombia.

Viveiros ATM, Godinho HP. Sperm quality and criopreservation of Brazilian freshwater fish species: a review. Fish Physiol Biochem. 2009;35(1):137-50.

Viveiros ATM, Fessehaye $\mathrm{Y}$, ter Veld M, Schulz RW, Komen J. Hand-stripping of semen and semen quality after maturational hormone treatments, in African catfish Clarias gariepinus. Aquaculture. 2002; 213:373-386.

Viveiros ATM, Nascimento AF, Orfão LH, Isaú ZA. Motility and fertility of the subtropical freshwater fish streaked prochilod (Prochilodus lineatus) sperm cryopreserved in powdered coconut water. Theriogenology. 2010;(74-4):551-556.

Zohar Y, Mylonas Constantinos C. Endocrine manipulations of spawning in cultured fish: from hormones to genes. Aquaculture. 2001;197:99-136. 